\title{
VECTORES DE LA LEISHMANIASIS TEGUMENTARIA Y LA ENFERMEDAD DE CARRIÓN EN EL PERÚ: UNA ACTUALIZACIÓN
}

\author{
Victor Zorrilla1,2,a , Gissella Vásquez ${ }^{1, b}$, Liz Espada1,c, Pablo Ramírez²,d
}

\begin{abstract}
RESUMEN
De las aproximadamente 190 especies de Lutzomyia en el Perú, solo un reducido número han sido incriminadas como vectores de leishmaniasis tegumentaria en valles occidentales e interandinos: Lutzomyia ( $L u$.) peruensis, Lu. verrucarum, Lu. tejadai, Lu. ayacuchensis, Lu. pescei; mientras que en la región amazónica Lu. yuilli yuilli, Lu. chagasi, Lu. davisi y $L u$. auraensis han sido encontradas infectadas naturalmente con Leishmania del subgénero Viannia. Lutzomyia auraensis constituye un nuevo reporte como vector potencial de leishmaniasis en regiones neotropicales. En relación a los vectores de la bartonellosis humana o enfermedad de Carrión, Lu. verrucarum y Lu. peruensis son los vectores principales y de más amplia distribución en regiones andinas del norte, centro y sur del Perú. Otros potenciales vectores de la enfermedad de Carrión son Lu. serrana en el valle del Monzón, Huamalíes, Huánuco, Lu. pescei en Apurímac y Cusco; Lu. robusta y Lu. maranonensis en las provincias de Jaén, San Ignacio y Utcubamba, selva alta del Perú. Debido a la alta prevalencia de la leishmaniasis y bartonellosis en el Perú y a su dispersión que sobrepasa los límites de las áreas endémicas conocidas, es necesario actualizar tanto la información como los mapas de distribución de los vectores de estas enfermedades, para contribuir con el mejoramiento de las medidas de prevención y control. La información existente sobre flebotomíneos vectores en el Perú ha sido reunida en este artículo.
\end{abstract}

Palabras clave: Lutzomyia; Leishmaniasis; Bartonellosis; Vectores; Perú (fuente: DeCS BIREME).

\section{UPDATE ON TEGUMENTARY LEISHMANIASIS AND CARRION'S DISEASE VECTORS IN PERU}

\begin{abstract}
Among approximately 190 species of Lutzomyia in Peru, only a small number have been identified as vectors of tegumentary leishmaniasis in Western and inter-Andean valleys. These include $L$. peruensis, $L$. verrucarum, $L$. tejadai, $L$. ayacuchensis, and $L$. pescei. In the Amazon region, $L$. yuilli yuilli, L. chagasi, $L$. davisi, and $L$. auraensis are naturally infected, among the subgenera Leishmania and Viannia. L. auraensis is newly reported as a potential vector of leishmaniasis in neotropical regions. Among the primary and most widely distributed vectors of human bartonellosis or Carrión's disease, $L$. verrucarum and $L$. peruensis are predominant in the Andean regions of northern, central, and southern Peru. Other potential vectors of Carrion's disease are L. serrana in the Monzon Valley, Huamalies, and Huanuco; L. pescei in Apurímac and Cusco; and $L$. robusta and $L$. maranonensis in Jaén, San Ignacio, and Utcubamba provinces, and the high forests of Peru. Because of the high prevalence of leishmaniasis and bartonellosis outside of known endemic areas in Peru, it is necessary to update data and distribution maps of these disease vectors. This may improve both prevention and control measures. Existing information about sandfly vectors in Peru is also provided in this article.
\end{abstract}

Key words: Lutzomyia; Leishmaniasis; Bartonellosis; Vectors; Peru (source: MeSH NLM).

\section{INTRODUCCIÓN}

Los flebotomíneos son pequeños insectos del orden Diptera, familia Psychodidae, subfamilia Phlebotominae, con más de 800 especies descritas agrupadas, hasta hace poco, en dos géneros de importancia médica, Phlebotomus en el Viejo Mundo y Lutzomyia en América ${ }^{(1,2)}$. Recientemente, Warileya rotundipennis fue encontrada infectada naturalmente con Leishmania (Viannia) en un foco de leishmaniasis cutánea de la Municipalidad de

\footnotetext{
Departamento de Entomología, Centro de Investigación de Enfermedades Tropicales de la Marina de los EE. UU. (NAMRU-6). Lima, Perú.

Facultad de Ciencias Biológicas, Universidad Nacional Mayor de San Marcos. Lima, Perú.

Biólogo; ${ }^{\mathrm{b}}$ Ph.D. en Entomología; ${ }^{\mathrm{c}}$ Bachiller en Biología; ${ }^{\mathrm{d}}$ Doctor en Ciencias

Este trabajo es parte de la tesis de posgrado en Biología Molecular (Facultad de Ciencias Biológicas, Universidad Nacional Mayor de San Marcos) de uno de los autores (V. Zorrilla)

Recibido: 19/09/2016 Aprobado: 12/07/2017 En Línea: 29/09/2017
}

Citar como: Citar como: Zorrilla V, Vásquez G, Espada L, Ramírez P. Vectores de la leishmaniasis tegumentaria y la Enfermedad de Carrión en el Perú: una actualización. Rev Peru Med Exp Salud Publica. 2017;34(3):485-96.doi: 10.17843/rpmesp.2017.343.2398 
Puerto Rico, departamento de Risaralda, Colombia, en la vertiente occidental de los Andes, con lo que se eleva a tres los géneros de flebotomíneos que tienen importancia médica ${ }^{(3)}$. En la región Neotropical se distribuyen desde el sur de los Estados Unidos hasta el norte de Argentina $\mathrm{y}$, además de transmitir parásitos del género Leishmania, algunas especies son vectores de Arbovirus (género Flevovirus) y de la bacteria Bartonella bacilliformis ${ }^{(4)}$. Se desconoce cuándo hicieron su aparición en el mundo, pero se asume que ocurrió a finales del Paleozoico y principios del Mesozoico ${ }^{(5)}$. Por otro lado, el restringido número de especies con capacidad de transmitir Leishmania sugiere que deben existir condiciones especiales de adaptación evolutiva entre el patógeno y el vector, que permitan el desarrollo del ciclo biológico del patógeno en el tracto digestivo del flebotomíneo y que a su vez estos sean capaces de transmitirlo por picadura ${ }^{(6)}$. En el caso de $B$. bacilliformis, la coevolución con sus vectores, que incluye un restringido número de especies del género Lutzomyia en el Perú, Ecuador y Colombia, es mucho menos conocida, puesto que hasta ahora el único reservorio conocido de $B$. bacilliformis es el ser humano, a diferencia de las otras especies de Bartonella que muestran una mayor diversificación en relación a sus vectores y hospederos ${ }^{(7-9)}$.

Para la clasificación taxonómica de los flebotomíneos existen dos modelos sistemáticos, el de Young \& Duncan $(1994)^{(1)}$ y el propuesto por Eunice Galati (1995-2003) ${ }^{(4)}$, los que son usados actualmente por diferentes grupos de investigadores. Para mayor claridad, en esta revisión bibliográfica se utilizará el modelo sistemático de Young \& Duncan (1994) (1). Hasta el 2001 habían sido reportadas 149 especies de flebotomíneos en el Perú ${ }^{(10)}$, pero en la actualidad se considera que existen más de 190 especies (Departamento de Entomología, Centro de Investigación de Enfermedades Tropicales de la Marina de los EE. UU., datos no publicados), de las cuales el $80 \%$ se distribuyen en la selva amazónica. Las especies del género Lutzomyia en el Perú, de acuerdo a la clasificación de Young \& Duncan (1994) ${ }^{(1)}$ pertenecen a los subgéneros Lutzomyia, Sciopemyia, Pintomyia, Pressatia, Evandromyia, Viannamyia, Psathyromyia, Nyssomyia, Trichophoromyia, Psychodopygus, Pilosa, Trichopygomyia, Micropygomyia y Helcocyrtomyia, y a los grupos Migonei, Verrucarum, Saulensis, Baityi, Aragaoi, Dreisbachi y Oswaldoi (11).

El área de distribución espacial y altitudinal de los flebotomíneos supera ampliamente el de las enfermedades que transmiten, pero estas no pueden existir sin los vectores $^{(12,13)}$. En el Perú, los flebotomíneos se distribuyen desde aproximadamente $900 \mathrm{~m}$ de altitud hasta altitudes superiores a los $3500 \mathrm{~m}$ en los valles occidentales e interandinos, limitado, principalmente, por la temperatura y el tipo de vegetación presente ${ }^{(14-17)}$, y en toda la selva amazónica, por debajo de los $2000 \mathrm{~m}$ de altitud ${ }^{(18-20)}$.

En el Perú, los flebotomíneos son conocidos con una gran variedad de nombres regionales. En la selva y los valles orientales se les conoce como "manta blanca", "espundia", "rapacha", "k'ete" o "wanwa", "karachupa usa"; en la sierra adoptan diferentes nombres, según la región: "uta", "titiras" (sierra central), "angelillos" (Huánuco), "capa blanca" (Ayacucho), "puma”, "pumamanchachi”, "pumakanikum" y "chuspi” (Ancash), "alalapo" (norte de Cajamarca) ${ }^{(11,17,21,22) .}$

La determinación de una especie de flebotomíneo como vector de leishmaniasis o bartonellosis se basa en una serie de criterios ${ }^{(6,17)}$. Los vectores comprobados son aquellos que permiten el desarrollo del ciclo biológico del agente patógeno y pueden transmitirlo por picadura a un hospedero susceptible ${ }^{(23)}$, mientras que un vector incriminado o potencial es aquel en el que solo se ha llegado a determinar la infección natural con el agente patógeno, ya sea por métodos moleculares, el aislamiento del patógeno por disección del tracto digestivo, o una combinación de ambas técnicas ${ }^{(24,25)}$. Algunos vectores son específicos para una sola especie de patógeno (Ej. Phlebotomus papatasi solo transmite L. major en el Viejo Mundo) ${ }^{(26)}$, mientras que los vectores permisivos son los que tienen capacidad de transmitir diferentes especies del mismo patógeno (Ej. Lu. migonei puede transmitir $L$. braziliensis y $L$. infantum) ${ }^{(27)}$, o patógenos de diferentes especies $(\mathrm{Ej}$. Lu. peruensis puede transmitir Leishmania y Bartonella) ${ }^{(10)}$.

En la actualidad, la biología molecular se ha convertido en la principal herramienta para incriminar potenciales vectores de diversos patógenos, siendo la reacción en cadena de la polimerasa (PCR) una de las más utilizadas pues permite analizar un gran número de muestras en un corto periodo, lo que representa una gran ventaja sobre el método de disección, el cual requiere personal entrenado y consume mucho tiempo ${ }^{(28)}$. Además de la detección del ADN de Leishmania en el potencial vector por PCR, el reciente hallazgo de receptores de lipofosfoglicanos (LPG) de Leishmania en el tracto digestivo de algunas especies de flebotomíneos por técnicas bioquímicas y moleculares es un importante aporte en la determinación de su rol vectorial, pues se sabe que la unión de Leishmania a estos receptores LPG es crucial para evitar la acción de las enzimas digestivas y permitir el desarrollo del parásito en el vector ${ }^{(6,26)}$

Las enfermedades transmitidas por flebotomíneos siguen teniendo gran impacto sobre la salud pública en el Perú, debido a su alta prevalencia y a su dispersión hacia otras regiones donde previamente no se habían registrado casos de leishmaniasis y bartonellosis ${ }^{(29)}$. El conocimiento actual sobre los vectores o potenciales vectores de estas enfermedades es limitado, por lo que es necesario actualizar la información que se tiene sobre los flebotomíneos de importancia médica en el Perú, para lo cual se ha hecho una revisión exhaustiva de todas las publicaciones existentes sobre el tema con la finalidad de reunir y consolidar esta información de tipo narrativa en un solo artículo. 


\section{ENFERMEDADES TRANSMITIDAS POR FLEBOTOMÍNEOS EN EL PERÚ}

\section{LEISHMANIASIS TEGUMENTARIA}

La leishmaniasis es causada por un protozoo parásito intracelular del género Leishmania (Kinetoplastida, Trypanosomatidae), y es endémica en 98 países tropicales y subtropicales, donde más de 350 millones de personas están en riesgo, y se estima que cada año se presentan 2 millones de casos nuevos, de los cuales 500 mil corresponden a leishmaniasis visceral ${ }^{(30)}$. Las especies de Leishmania patógenas para el ser humano pertenecen a dos subgéneros, Viannia y Leishmania; la evolución y severidad de la enfermedad está en relación directa a la especie de Leishmania infectante y a la respuesta inmune del hospedero ${ }^{(31,32)}$. Con pocas excepciones, las leishmaniasis son zoonosis, el parásito tiene una amplia variedad de mamíferos reservorios silvestres y algunos domésticos ${ }^{(33)}$. La infección en los reservorios es inaparente, salvo en los perros quienes presentan lesiones cutáneas al igual que el hombre, o pueden hacer una infección visceral generalizada de consecuencias fatales $\sin$ tratamiento adecuado ${ }^{(34,35)}$.

La leishmaniasis es una de las diez enfermedades más desatendidas desde el punto de vista de la salud pública, y puede llegar a ser una enfermedad muy incapacitante y deformante teniendo un serio impacto sobre la economía, tanto de la población expuesta como para los servicios de salud por el alto costo que demanda el tratamiento ${ }^{(21,36)}$. La leishmaniasis visceral puede llegar a ser mortal, especialmente en pacientes que no han recibido tratamiento e, incluso en los casos tratados la tasa de mortalidad varía entre el 10 al $20 \%{ }^{(37)}$.

En el Perú se conocen dos formas de leishmaniasis de importancia epidemiológica: la leishmaniasis andina, endémica en valles occidentales e interandinos del norte y centro entre los 1000 y 3200 m de altitud, y la leishmaniasis selvática, por debajo de los 1800 m de altitud ${ }^{(21,38)}$ (Figura 1). Los límites altitudinales de las áreas de transmisión pueden ampliarse o reducirse de acuerdo a las condiciones climáticas ("halo epidémico") (12), y cada forma de leishmaniasis tiene un patrón epidemiológico y modo de transmisión diferente, relacionado con el comportamiento humano, la presencia de reservorios, el comportamiento de los flebotomíneos y la especie de Leishmania circulante en cada zona ${ }^{(39-41)}$.

En la región andina del Perú predomina la leishmaniasis cutánea causada por Leishmania (Viannia) peruviana, mientras que en la selva la leishmaniasis cutáneomucosa causada por Leishmania (Viannia) braziliensis constituye un serio problema de salud, causada por la metástasis del parásito a partir de una lesión cutánea inicial hacia la mucosa nasoorofaringea, y que se presenta en un 20$40 \%$ de pacientes ${ }^{(21,36)}$. Otras especies de Leishmania encontradas en la selva peruana son: Leishmania (Viannia) lainsoni, L. (V.) guyanensis, L. (V.) shawi y L. (Leishmania) amazonensis ${ }^{(42,43)}$. Hasta el momento no se han reportado casos de leishmaniasis visceral en nuestro país, tampoco se tiene referencias de la presencia de su principal vector, Lutzomyia longipalpis ${ }^{(10)}$.

La probabilidad de que la leishmaniasis andina y selvática coexistan en determinadas regiones siempre ha estado presente, y esto ha sido observado en el valle interandino del Huallaga, provincia de Ambo, Huánuco, que por su ecología es un valle abierto hacia la selva amazónica. En esta área, durante los años 80 y 90 se observó un incremento inusual en el número de casos de leishmaniasis ${ }^{(44)}$. Estudios posteriores determinaron la coexistencia de $L$. braziliensis y $L$. peruviana en la zona, y se reporta por primera vez el aislamiento de híbridos putativos $L$. braziliensis / $L$. peruviana en algunos pacientes ${ }^{(45)}$, lo que sugiere un incremento del riesgo de metástasis hacia las mucosas ${ }^{(45)}$. Estos importantes hallazgos fueron complementados recientemente con la detección de un híbrido $L$. braziliensis / L. peruviana en Lutzomyia tejadai, el principal vector de la leishmaniasis en Huánuco ${ }^{(46)}$.

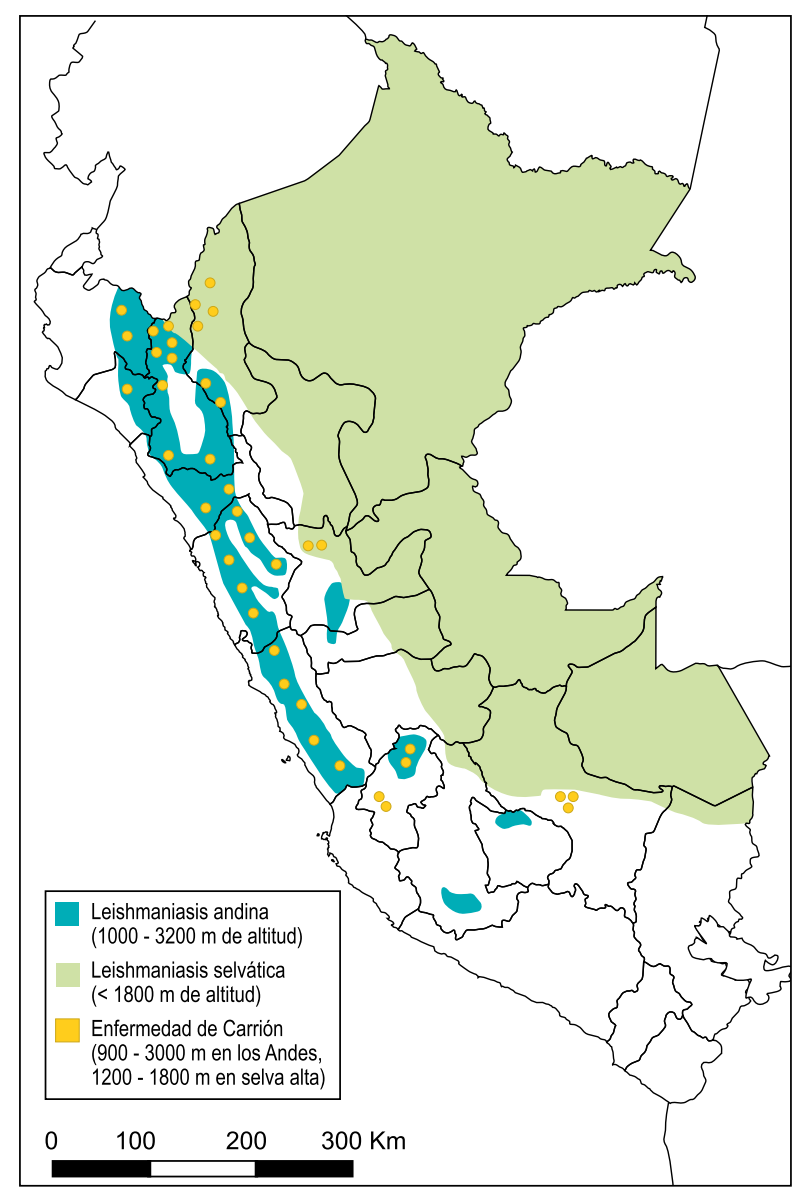

Figura 1. Áreas de riesgo para la transmisión de la leishmaniasis y la enfermedad de Carrión en el Perú 


\section{BARTONELLOSIS HUMANA O ENFERMEDAD DE CARRIÓN}

La bartonellosis humana, también llamada enfermedad de Carrión, "verruga peruana" o "fiebre de la Oroya", es causada por una bacteria pleomórfica Gram negativa, intracelular, Bartonella bacilliformis, y es una enfermedad grave que en su forma febril anemizante presenta alta tasa de mortalidad (mayor al $85 \%$ ) si el paciente no recibe tratamiento antibacteriano adecuado ${ }^{(47)}$.

La bartonellosis comparte las mismas áreas endémicas con la leishmaniasis tegumentaria en las zonas andinas del norte, centro y la región nororiental del Perú (Figura 1), en raras ocasiones puede haber coinfección con ambas enfermedades en un mismo paciente ${ }^{(39)}$. La bacteria parasita los glóbulos rojos (parasitemia del 1 al 100\%) causando anemia severa ${ }^{(47,48)}$. Los que sobreviven a la infección aguda, después de un periodo intermedio de duración variable, desarrollarán la clásica presentación eruptiva con verrugas en la piel, muy vascularizadas, de diferente tamaño y en número variable ${ }^{(47)}$. En zonas endémicas de Ancash y el Norte del Perú las formas eruptivas son frecuentes, mientras que en la selva central (Huánuco) y el Sur (Urubamba, Cusco), es la presentación aguda la que predomina ${ }^{(49,50)}$.

Durante la construcción del ferrocarril central Lima-La Oroya, fallecieron más de 7000 obreros en el valle del Rímac, víctimas de una enfermedad mortal llamada "fiebre de la Oroya", de origen desconocido en esa época. Investigaciones posteriores pudieron determinar que el agente causal era una bacteria, a la que se denominó Bartonella bacilliformis en honor a su descubridor, Alberto Barton (48).

La dificultad para reproducir la infección por $B$. bacilliformis en animales de experimentación es uno de los principales obstáculos para comprender mejor esta enfermedad. Desde el famoso experimento realizado por Daniel A. Carrión, quien se autoinoculó material obtenido de un verrucoma de un paciente con fase eruptiva, para demostrar que la "verruga peruana" y la fiebre de la Oroya eran dos presentaciones clínicas diferentes de la misma enfermedad, hasta la actualidad ninguno de los investigadores que intentaron replicar el experimento de Carrión en ellos mismos o en "voluntarios", han podido reproducir el cuadro clínico febril y anemizante de la bartonellosis ${ }^{(51)}$.

\section{VECTORES DE LA LEISHMANIASIS TEGUMENTARIA EN EL PERÚ}

La asociación entre la leishmaniasis tegumentaria y los flebotomíneos como transmisores del agente etiológico de esta enfermedad, fue descrita por Cosme Bueno en 1764, quien menciona que la picadura de un pequeño insecto al que los nativos llamaban "uta", producía lesiones corrosivas en la piel de muy difícil tratamiento ${ }^{(52)}$. En las primeras décadas del siglo $\mathrm{XX}$, se realizaron numerosos estudios acerca de la epidemiología y clínica de la leishmaniasis en el Perú (12), y posteriormente se fue conociendo mejor la biología y comportamiento de los flebotomíneos ${ }^{(13,14,53)}$, así como el desarrollo del ciclo biológico de algunas especies bajo condiciones de laboratorio ${ }^{(54)}$, pues en la naturaleza casi no se han encontrado estados inmaduros de Lutzomyia ${ }^{(1)}$.

\section{VECTORES DE LA LEISHMANIASIS ANDINA EN EL PERÚ}

Las investigaciones realizadas hasta el momento han permitido determinar que los vectores de la leishmaniasis andina son (Tabla 1, Figura 2):

Lutzomyia (Helcocyrtomyia) peruensis. Es una de las especies predominantes en la región andina entre los 1000 y $3200 \mathrm{~m}$ de altitud, pero excepcionalmente supera estos límites, como en el valle del Vilcanota (Cusco) donde fue capturada a $3450 \mathrm{~m}$ de altitud ${ }^{(10,21)}$. Hasta el momento no se ha reportado su presencia en la región amazónica.

Lutzomyia verrucarum. Presenta amplia distribución en ambientes xerofíticos y subxerofíticos de los Andes del norte y centro del Perú, entre los 1000 y 3200 m de altitud ${ }^{(10)}$. No se ha reportado su presencia en la región amazónica.

Lutzomyia (Helcocyrtomyia) tejadai. Es la especie predominante en la región andina del valle del Huallaga y sus afluentes, departamento de Huánuco. Su distribución va desde los $3200 \mathrm{~m}$ de altitud en el valle interandino del río Huertas (Yanahuanca, Pasco) hasta los $800 \mathrm{~m}$ de altitud en la selva alta del valle del Monzón (Huánuco) ${ }^{(55)}$. Lu. tejadai es muy antropofílico y se le captura en alta densidad en el interior de las viviendas, incluso en zonas periurbanas de Ambo y Huánuco.

Lutzomyia (Helcocyrtomyia) ayacuchensis. Fue descrita a partir de ejemplares capturados en áreas endémicas de leishmaniasis de las provincias de Lucanas y Parinacochas (Ayacucho), entre los 1400 y los $2950 \mathrm{~m}$ de altitud (56). También se ha reportado su presencia en los departamentos de Ancash, La Libertad, Cajamarca y Piura ${ }^{(10,57)}$, así como en el sur de Ecuador (24).

Lutzomyia (Helcocyrtomyia) pescei. Es la especie predominante en las provincias de Andahuaylas (Apurímac) y Calca (Cusco), entre los 2200 y 3300 m de altitud, también ha sido capturada en Huancavelica ${ }^{(10)}$.

\section{POTENCIALES VECTORES DE LEISHMANIASIS EN LA REGIÓN AMAZÓNICA}

Son pocas las referencias acerca de la presencia de potenciales vectores de la leishmaniasis en la 
Tabla 1. Vectores y potenciales vectores de la leishmaniasis en el Perú

\begin{tabular}{|c|c|c|c|c|c|}
\hline $\begin{array}{l}\text { Especie de } \\
\text { Lutzomyia }\end{array}$ & $\begin{array}{l}\text { Especie de } \\
\text { Leishmania }\end{array}$ & $\begin{array}{c}\text { Método de incriminación } \\
\text { vectorial } \\
\end{array}$ & $\begin{array}{c}\text { Provincial } \\
\text { Departamento }\end{array}$ & $\begin{array}{c}\text { Area } \\
\text { geográfica }\end{array}$ & Referencias \\
\hline \multicolumn{6}{|l|}{ Región andina } \\
\hline Lu. peruensis & L. (V.) peruviana & Inoculación en hámster & Huarochirí (Lima) & $\begin{array}{c}\text { Valle } \\
\text { occidental }\end{array}$ & Herrer A. 1982 \\
\hline Lu. peruensis & L. (V.) peruviana & $\begin{array}{l}\text { Disección del tracto } \\
\text { digestivo, PCR }\end{array}$ & Huarochirí (Lima) & $\begin{array}{c}\text { Valle } \\
\text { occidental }\end{array}$ & Pérez et al. 2007 \\
\hline Lu. peruensis & L. (V.) peruviana & $\begin{array}{l}\text { Disección del tracto } \\
\text { digestivo }\end{array}$ & $\begin{array}{c}\text { Otuzco } \\
\text { (La Libertad) }\end{array}$ & $\begin{array}{c}\text { Valle } \\
\text { Interandino }\end{array}$ & Cruzado et al. 1997 \\
\hline Lu. peruensis & L. (V.)peruviana & Disección, PCR, isoenzimas & $\begin{array}{l}\text { Bolognesi } \\
\text { (Ancash) }\end{array}$ & $\begin{array}{c}\text { Valle } \\
\text { Interandino }\end{array}$ & Pérez et al. 1991 \\
\hline Lu. peruensis & L. (V.) peruviana & $\begin{array}{l}\text { PCR, Secuenciamiento de } \\
\text { ADN }\end{array}$ & $\begin{array}{c}\text { Otuzco } \\
\text { (La Libertad) }\end{array}$ & $\begin{array}{c}\text { Valle } \\
\text { Interandino }\end{array}$ & Córdova et al. 2011 \\
\hline Lu. verrucarum & L. (V.) peruviana & $\begin{array}{l}\text { Disección, PCR, } \\
\text { isoenzimas, transmisión } \\
\text { experimental en hámster }\end{array}$ & $\begin{array}{l}\text { Bolognesi } \\
\text { (Ancash) }\end{array}$ & $\begin{array}{c}\text { Valle } \\
\text { Interandino }\end{array}$ & $\begin{array}{l}\text { Pérez et al. 1991, } \\
\text { Davies et al. } 1993\end{array}$ \\
\hline Lu. ayacuchensis & L. (V.) peruviana & $\begin{array}{c}\text { Disección tracto digestivo, } \\
\text { PCR }\end{array}$ & $\begin{array}{l}\text { Lucanas y } \\
\text { Parinacochas } \\
\text { (Ayacucho) }\end{array}$ & $\begin{array}{c}\text { Valle } \\
\text { Interandino }\end{array}$ & Cáceres et al. 2004 \\
\hline Lu. ayacuchensis & L. (V.) guyanensis & $\begin{array}{l}\text { PCR, Secuenciamiento de } \\
\text { ADN }\end{array}$ & $\begin{array}{c}\text { Otuzco } \\
\text { (La Libertad) }\end{array}$ & $\begin{array}{c}\text { Valle } \\
\text { Interandino }\end{array}$ & Córdova et al. 2011 \\
\hline Lu. tejadai & $\begin{array}{l}\text { L. (V.) braziliensis / L. } \\
\text { (V.) peruviana (híbrido) }\end{array}$ & $\begin{array}{l}\text { PCR, Secuenciamiento de } \\
\text { ADN }\end{array}$ & Ambo (Huánuco) & $\begin{array}{c}\text { Valle } \\
\text { Interandino }\end{array}$ & Kato et al. 2016 \\
\hline Lu. pescei & L. (Viannia) spp. & $\begin{array}{c}\text { Correlación geográfica con } \\
\text { casos humanos }\end{array}$ & $\begin{array}{l}\text { Apurimac y } \\
\text { Cusco }\end{array}$ & $\begin{array}{c}\text { Valles } \\
\text { Interandinos }\end{array}$ & $\begin{array}{l}\text { Cáceres \& Galati } \\
2001\end{array}$ \\
\hline \multicolumn{6}{|l|}{$\begin{array}{l}\text { Región } \\
\text { amazónica }\end{array}$} \\
\hline Lu. yuilli yuilli & L.(Viannia) spp. & PCR & $\begin{array}{c}\text { Echarati, La } \\
\text { Convención } \\
\text { (Cusco) }\end{array}$ & Selva Alta & Paucar R. 2001 \\
\hline Lu. chagasi & L. (Viannia) spp. & Disección, cultivo, PCR & $\begin{array}{l}\text { Echarati, La } \\
\text { Convención } \\
\text { (Cusco) }\end{array}$ & Selva Alta & Paucar R. 2001 \\
\hline Lu. auraensis & L. (V.) braziliensis & FRET Real Time PCR & $\begin{array}{c}\text { Iberia } \\
\text { (Madre de Dios) }\end{array}$ & Selva Baja & Valdivia et al. 2012 \\
\hline Lu. auraensis & L. (V.) lainsoni & FRET Real Time PCR & $\begin{array}{c}\text { Iberia } \\
\text { (Madre de Dios) }\end{array}$ & Selva Baja & Valdivia et al. 2012 \\
\hline Lu. davisi & L. (V.) braziliensis & FRET Real Time PCR & $\begin{array}{c}\text { Iberia } \\
\text { (Madre de Dios) }\end{array}$ & Selva Baja & Valdivia et al. 2012 \\
\hline
\end{tabular}

selva peruana ${ }^{(58)}$, y la mayoría de los conocimientos actuales provienen de estudios realizados en otros países amazónicos, principalmente Brasil, Colombia, Ecuador y Bolivia.

En el Perú, Paucar (2001) ${ }^{(59)}$ determinó la infección natural de Lutzomyia (Nyssomyia) yuilli yuilli y Lutzomyia (Psychodopygus) chagasi con Leishmania (Viannia) spp. en Echarati, provincia de La Convención (Cusco). Recientemente Valdivia et al. (2012) ${ }^{(60)}$ determinaron la infección natural de Lutzomyia (Trichophoromyia) auraensis con Leishmania (V.) braziliensis y Leishmania (V.) lainsoni; y Lutzomyia (Psychodopygus) davisi con Leishmania (V.) braziliensis. Lutzomyia yuilli yuilli está distribuida en áreas de selva alta y baja de los departamentos de Madre de Dios, Cusco, Junín, Huánuco, Loreto y Amazonas. Lu. chagasi,
$L u$. davisi y Lu. auraensis se distribuyen principalmente en la selva baja y algunas regiones de selva alta, desde Loreto hasta Madre de Dios y Puno (Figura 3$)^{(18,19,22)}$.

\section{CRITERIOS DE INCRIMINACIÓN DE VECTORES DE LEISHMANIASIS}

Los criterios más importantes para considerar vector de Leishmania a una determinada especie de flebotomino son: a) El vector tiene que ser antropofílico, es decir, picar al ser humano; b) debe ser zoofílico, es decir debe picar a animales domésticos y silvestres; c) al vector se le debe encontrar infectado naturalmente con la misma especie de Leishmania encontrada en el hospedero; d) el vector debe favorecer el desarrollo del parásito en su tubo digestivo y ser capaz de transmitirlo al hospedero 


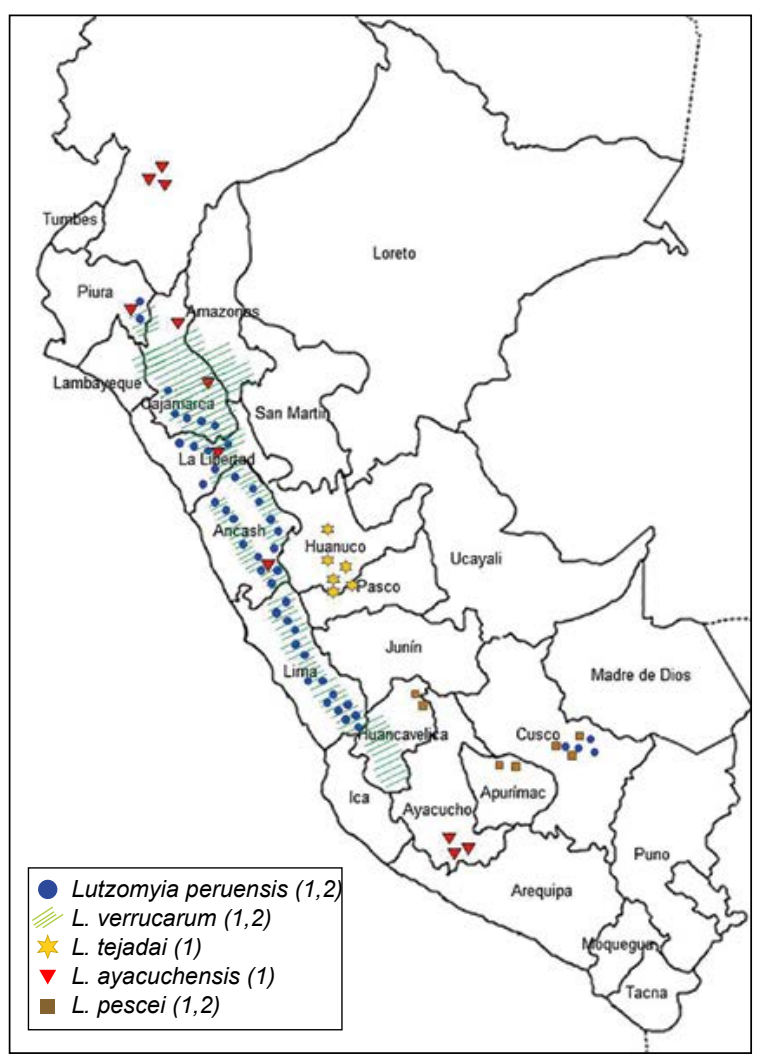

Figura 2. Distribución geográfica de vectores y potenciales vectores de leishmaniasis (1) y la enfermedad de Carrión (2) en valles occidentales e interandinos del Perú (Modificado de Cáceres \& Galati 2001)

por picadura; e) la distribución geográfica del vector debe coincidir con la de la enfermedad que transmite, observando además una fuerte asociación ecológica, incluyendo estacionalidad, entre las tasas de densidad del vector, la infección humana y los reservorios; f) se debe demostrar que una especie de vector es esencial para el mantenimiento de la transmisión de la leishmaniasis, con o sin la participación de otras especies; y g) se debe observar un decrecimiento significativo en la incidencia de la enfermedad en relación a un decrecimiento también significativo en la densidad del vector después de la aplicación de las medidas de control ${ }^{(6,17)}$.

De todos los criterios expuestos, los dos primeros (antropofilia y zoofilia) han sido bien determinados en la mayoría de especies conocidas de flebotomíneos vectores en el Perú, y la correlación geográfica entre el vector y la enfermedad sigue siendo uno de los más utilizados para incriminar vectores en nuestro país y en otras regiones del mundo. Respecto a los otros criterios, se tienen muy pocas referencias.

\section{CORRELACIÓN GEOGRÁFICA DEL VECTOR CON LA ENFERMEDAD}

Los focos de transmisión de la leishmaniasis corresponden a regiones naturales geográficas que por sus características ecológicas, climáticas y altitudinales permiten la presencia de flebotomíneos hematófagos. Esto significa que la distribución de casos de leishmaniasis en una determinada área geográfica se superpone a la distribución de los flebotomíneos potenciales vectores ${ }^{(13)}$. Este criterio sigue siendo utilizado con frecuencia en la actualidad, y por el momento es la única evidencia de que Lutzomyia pescei es el vector potencial de Leishmania en las provincias de Andahuaylas (Apurímac) y Calca (Cusco) ${ }^{(10)}$, y Lutzomyia robusta en el sur de Ecuador ${ }^{(61)}$.

\section{INFECCIÓN NATURAL DE LUTZOMYIA SPP. CON LEISHMANIA}

Uno de los primeros trabajos publicados para demostrar experimentalmente que una especie de Lutzomyia es vector de Leishmania fue el realizado por Herrer (1982) ${ }^{(62)}$. Este investigador preparó triturados de ejemplares hembras de Lutzomyia peruensis capturadas en los valles de Santa Eulalia y Tapicara (Huarochirí, Lima), los que fueron inoculados por vía intradérmica en la nariz de hámster (Mesocricetus auratus) machos. El examen microscópico determinó que dos de siete hámsters inoculados se infectaron por Leishmania. Este trabajo sivió para demostrar que Lutzomyia peruensis es el vector natural de la leishmaniasis tegumentaria en el Perú.

Otra técnica utilizada para determinar la infección natural de flebotomíneos con Leishmania es la disección del tracto digestivo. La ventaja del método de disección es que permite el aislamiento del parásito vivo, pero la desventaja es que requiere un examen inmediato del tracto digestivo de los flebotomíneos en el campo, o transportar los insectos en nitrógeno líquido hasta el laboratorio donde se realizará el estudio. El procedimiento consume mucho tiempo y requiere de personal bien entrenado ${ }^{(28)}$. La técnica de disección permitió determinar la infección natural de Lutzomyia ayacuchensis con Leishmania peruviana y reportar a este flebotomino como nuevo vector de leishmaniasis en Lucanas y Parinacochas (Ayacucho). Fueron disectados 1849 ejemplares hembras de Lutzomyia ayacuchensis, aislando el parásito de cinco de ellas (tasa de infección natural del $0,3 \%$ ), el que posteriormente fue caracterizado como Leishmania (Viannia) peruviana por cariotipaje molecular ${ }^{(56)}$. Lutzomyia ayacuchensis también ha sido encontrado infectado naturalmente con Leishmania spp. en el sur de Ecuador (24). Pérez et al. (2007) ${ }^{(25)}$, en la localidad de Chaute, provincia de Huarochirí, Lima, determinaron la infección natural de Lutzomyia peruensis con Leishmania (Viannia) peruviana (1 de 75 ejemplares, 
$1,3 \%)$ por disección del tubo digestivo, confirmando los resultados por biología molecular (PCR).

\section{INFECCIÓN EXPERIMENTAL DE LUTZOMYIA CON LEISHMANIA EN EL LABORATORIO}

En el Perú, Davies et al. (1993) (23) demostraron por primera vez la transmisión de Leishmania peruviana por Lutzomyia verrucarum en un experimento bajo condiciones de laboratorio. Hámsters previamente infectados con Leishmania peruviana sirvieron como fuente de alimentación sanguínea a Lutzomyia verrucarum obtenidas de colonia; dos semanas después estos mismos flebotomíneos fueron alimentados sobre hamsters libres de infección por Leishmania, y se observó que uno de ellos desarrolló la enfermedad. Los resultados sugieren que no todos los individuos de una misma especie de flebotomíneo poseen la capacidad para infectarse con el parásito y poder transmitirlo por picadura (solo 1 Lutzomyia de un total de $59,1,7 \%$ ), y que posiblemente esta capacidad vectorial limitada dentro de la misma especie esté en relación directa a la variabilidad genética intraespecífica que presentan los flebotomíneos ${ }^{(6,63)}$.

\section{HERRAMIENTAS DE BIOLOGÍA MOLECULAR PARA LA DETERMINACIÓN DE LOS VECTORES DE LA LEISHMANIASIS}

El uso de la biología molecular para la detección de Leishmania en Lutzomyia es relativamente reciente, siendo la reacción en cadena de la polimerasa (PCR) la más utilizada por su alta sensibilidad y especificidad, además que permite el análisis de un gran número de muestras en un corto periodo ${ }^{(28)}$.

Las técnicas más utilizadas para la detección de Leishmania se basan en la amplificación de las regiones conservadas del ADN de los minicírculos presentes en el kinetoplasto del parásito. Una de estas técnicas es la descrita por López et al. (1993) ${ }^{(64)}$, originalmente diseñada para el diagnóstico de leishmaniasis en muestras clínicas de pacientes utilizando los primers MP1-L y MP3-H, y fue aplicado con éxito por Pérez et al. (1994) ${ }^{(65)}$ para la detección de Leishmania del subgénero Viannia en Lutzomyia peruensis y Lutzomyia verrucarum, procedentes de la localidad de Chaute (Huarochirí, Lima). Utilizando esta misma técnica, Páucar (2001) ${ }^{(59)}$ determinó la infección natural de Lutzomyia yuilli yuilli y Lutzomyia chagasi con Leishmania del subgénero Viannia, en el distrito de Echarati, provincia de La Convención, Cusco. Otro método para determinar la infección natural de Lutzomyia con Leishmania es el descrito por Kato et al. (2005) ${ }^{(28)}$. Los primers L.MC.1S y L.MC.1R amplifican regiones conservadas del ADN del kinetoplasto de Leishmania spp., mientras que los primers L.cyt.S y L.cyt.R amplifican una región del gen cyt $B$ (citocromo B) de Leishmania, lo que permite su clonamiento y posterior secuenciamiento para determinar la especie del parásito infectante ${ }^{(28)}$. Con esta técnica se determinó la infección natural de Lutzomyia peruensis con Leishmania (Vianna) peruviana en el valle de Huayllacayán (Ancash) y Otuzco (La Libertad) ${ }^{(66)}$, y de Lutzomyia ayacuchensis con Leishmania (Viannia) guyanensis en Otuzco ${ }^{(57)}$.

Recientemente, Kato et al. (2016) ${ }^{(46)}$ describen por primera vez la infección natural de Lutzomyia tejadai con un híbrido Leishmania (Viannia) braziliensis / Leishmania (V.) peruviana en el valle interandino del río Huallaga, Huánuco. Este híbrido fue previamente descrito por Dujardin et al. $(1995){ }^{(45)}$, a partir de un cultivo aislado de un paciente de Huancapallac, Huánuco, y sería el responsable de la leishmaniasis cutánea y cutáneomucosa en la región andina de Huánuco.

Utilizando PCR en tiempo real, Valdivia et al. (2012) (60) determinaron por primera vez la infección natural de Lutzomyia auraensis con Leishmania braziliensis y Leishmania lainsoni en Flor de Acre (Iberia, Madre de Dios). La técnica utilizada, Fluorescence Resonance Energy Transfer-Based Real-Time Polymerase Chain Reaction (FRET Based RT-PCR), consiste en un Nested-PCR para detectar mutaciones conocidas de los genes MPI y 6PGD que producen temperaturas de fusión (melting) distintos, y permite identificar diferentes especies de Leishmania. En este estudio también se determinó la infección natural de Lutzomyia davisi con Leishmania braziliensis.

La elevada sensibilidad del Nested RT-PCR permite detectar menos de cinco parásitos por muestra $(<60$ femtogramos de $A D N$ ), y es muy específica para identificar todas las especies de Leishmania presentes en Perú, lo que representa una gran ventaja frente al método de disección del tracto digestivo, que consume mucho tiempo, requiere de personal bien entrenado y además presenta baja especificidad pues los flebotomíneos podrían estar infectados con otros tripanosomatídeos diferentes de Leishmania ${ }^{(67,68)}$.

La tasa de infección natural de un flebotomíneo con Leishmania se obtiene de dividir el total de positivos sobre el total de muestras analizadas, pero hay que tener en cuenta si las hembras de flebotomíneos fueron analizadas en forma individual o se agruparon en pooles, lo que representa un sesgo en el análisis de datos. Por ejemplo, Kato et al. (2016) ${ }^{(46)}$ encontró solo 01 Lu. tejadai infectada con un híbrido $L$. braziliensis / L. peruviana de un total de 2997 especímenes analizados individualmente (tasa de infección natural $=0,03 \%$ ), mientras que en el trabajo reportado por Valdivia et al. (2012) ${ }^{(60)}, 1299$ hembras de Lutzomyia fueron agrupadas en 164 pooles (1-10 individuos/pool), de los cuales 7 fueron positivos a Leishmania. El análisis estadístico con intervalos de confianza al $95 \%$ estima que la prevalencia mínima de infección para $L u$. auraensis y $L u$. davisi fue de 0,6\% $(0,20$ 
- 1,42) y $0,9 \%(0,02-5,10)$ respectivamente ${ }^{(60)}$, puesto que en un pool positivo de 10 flebotomíneos se desconoce cuántos de ellos podrían estar infectados.

\section{DETERMINACIÓN DE LOS VECTORES DE LA ENFERMEDAD DE CARRIÓN}

Desde el famoso experimento de Daniel A. Carrión en 1885 , se han logrado importantes avances en la investigación clínica de la bartonellosis humana ${ }^{(47-49)}$, pero se desconocen importantes aspectos de la biología de la Bartonella bacilliformis en el vector, y la lista de vectores confirmados y potenciales es reducida (Tabla 2, Figura 3).

\section{LUTZOMYIA VERRUCARUM, PRINCIPAL VECTOR DE LA ENFERMEDAD DE CARRIÓN}

Las primeras evidencias con base científica de que Lutzomyia verrucarum es el vector de la bartonellosis humana fueron obtenidas en 1913, cuando el entomólogo norteamericano Charles Townsend capturó algunos ejemplares de esta especie en la estación del ferrocarril central de San Bartolomé, provincia de Huarochirí, Lima, donde habían ocurrido brotes frecuentes de la enfermedad de Carrión entre los obreros que trabajaban en la construcción del ferrocarril. Townsend asoció la presencia de este flebotomino con la transmisión de la enfermedad y describió a la especie como Phlebotomus verrucarum ${ }^{(48,69)}$. Entre los primeros trabajos experimentales para demostrar la infección natural de Lutzomyia verrucarum con Bartonella bacilliformis están los realizados por Hertig (1948) ${ }^{(53)}$, quien menciona la transmisión de la bacteria por picadura del insecto a monos Rhesus, y además da a conocer el aislamiento de $B$. bacilliformis en medio de cultivo a partir de flebotomíneos capturados en campo. Lutzomyia verrucarum tiene amplia distribución en áreas endémicas de bartonellosis de los valles occidentales e interandinos del norte y centro del Perú entre 1100 y 3200 metros de altitud, y su presencia sirve como criterio para determinar como zona verrucógena a una localidad donde este flebotomino está presente, pero se cree que existen otros vectores en zonas endémicas donde no se encuentra $L u$. Verrucarum ${ }^{(70)}$.

Ponce y Solórzano (2002) ${ }^{(71)}$ infectaron experimentalmente ejemplares de Lutzomyia verrucarum obtenidos de colonia con sangre infectada con $B$. bacilliformis procedentes de pacientes, para estudiar la posible transmisión transovárica de la bacteria en el vector. Del total de Lutzomyia alimentadas con sangre infectada, se detectó la infección con $B$. bacilliformis en el $8,3 \%$ por PCR, pero no hubo evidencia de transmisión transovárica.

La detección de la infección natural de Lutzomyia verrucarum con Bartonella bacilliformis por PCR en tiempo real fue realizada por Romero (2004) ${ }^{(72)}$ con una técnica modificada previamente descrita por Norman et al. (1995) ${ }^{(73)}$, diseñada para la detección de $B$. bacilliformis por amplificación del gen de la citratosintasa (gltA). Romero analizó 472 grupos de Lutzomyia verrucarum procedentes de Caraz, Ancash, de los cuales $13(2,75 \%)$ fueron positivos para $B$. bacilliformis, confirmados por secuenciamiento del ADN.

\section{LUTZOMYIA PERUENSIS, VECTOR DE LA ENFERMEDAD DE CARRIÓN EN CUSCO}

Durante 1997-1998 ocurrió un brote de bartonellosis humana en el Valle Sagrado de los Incas, Urubamba, Cusco ${ }^{(50)}$. Villaseca et al. (1999) ${ }^{(74)}$ realizaron un

Tabla 2. Vectores y potenciales vectores de la enfermedad de Carrión en el Perú

\begin{tabular}{|c|c|c|c|c|}
\hline $\begin{array}{l}\text { Especie de } \\
\text { Lutzomyia }\end{array}$ & Método de incriminación vectorial & $\begin{array}{c}\text { Provincia/ } \\
\text { Departamento }\end{array}$ & Área geográfica & Referencias \\
\hline Lu. verrucarum & $\begin{array}{c}\text { Correlación geográfica con casos } \\
\text { humanos }\end{array}$ & $\begin{array}{l}\text { Huarochirí } \\
\text { (Lima) }\end{array}$ & Valle occidental & Townsend 1913 \\
\hline Lu. verrucarum & $\begin{array}{l}\text { Aislamiento, } \\
\text { cultivo }\end{array}$ & $\begin{array}{l}\text { Huarochirí } \\
\text { (Lima) }\end{array}$ & Valle occidental & Hertig 1948 \\
\hline Lu. verrucarum & $\begin{array}{c}\text { Correlación geográfica con casos } \\
\text { humanos }\end{array}$ & $\begin{array}{c}\text { Norte y Centro del } \\
\text { Perú }\end{array}$ & $\begin{array}{l}\text { Valles Interandinos } \\
\text { y occidentales }\end{array}$ & Cáceres 1993 \\
\hline Lu. verrucarum & $\begin{array}{c}\text { PCR Tiempo Real, } \\
\text { secuenciamiento de ADN }\end{array}$ & $\begin{array}{l}\text { Caraz, Huaylas } \\
\quad \text { (Ancash) }\end{array}$ & Valle Interandino & Romero 2004 \\
\hline Lu. peruensis & $\begin{array}{c}\text { PCR, } \\
\text { secuenciamiento de ADN }\end{array}$ & $\begin{array}{l}\text { Urubamba } \\
\text { (Cusco) }\end{array}$ & Valle Interandino & Villaseca et al. 1999 \\
\hline Lu.maranonensis & $\begin{array}{c}\text { Correlación geográfica con casos } \\
\text { humanos }\end{array}$ & $\begin{array}{l}\text { Cajamarca y } \\
\text { Amazonas }\end{array}$ & Selva Alta & Cáceres et al. 1997 \\
\hline Lu. robusta & $\begin{array}{c}\text { Correlación geográfica con casos } \\
\text { humanos }\end{array}$ & $\begin{array}{l}\text { Cajamarca y } \\
\text { Amazonas }\end{array}$ & Selva Alta & Cáceres et al. 1997 \\
\hline Lu. serrana & $\begin{array}{c}\text { Correlación geográfica con casos } \\
\text { humanos }\end{array}$ & $\begin{array}{l}\text { Huamalíes } \\
\text { (Huánuco) }\end{array}$ & Selva Alta & Tejada et al. 2003 \\
\hline Lu. pescei & $\begin{array}{l}\text { Correlación geográfica con casos } \\
\text { humanos }\end{array}$ & $\begin{array}{l}\text { Huancavelica y } \\
\text { Cusco }\end{array}$ & $\begin{array}{l}\text { Valles Interandinos } \\
\text { y occidentales }\end{array}$ & Cáceres \& Galati 2001 \\
\hline
\end{tabular}




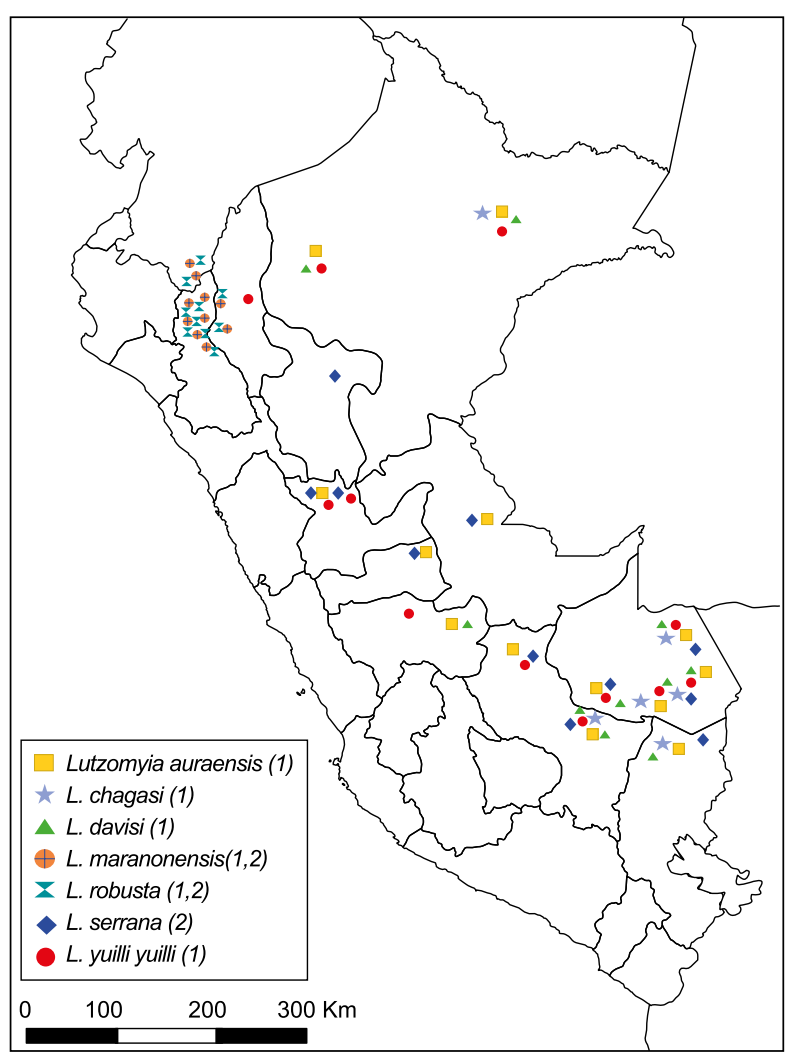

Figura 3. Distribución geográfica de vectores y potenciales vectores de leishmaniasis (1) y la enfermedad de Carrión (2) en áreas de selva alta y baja del Perú

estudio entomológico en la zona donde ocurrió el brote capturando Lutzomyia peruensis, única especie en la zona. De 104 especímenes analizados por PCR, en una se detectó $B$. bacilliformis y en otra Bartonella taylori, por secuenciamiento del ADN. Esta sería la primera evidencia de que Lutzomyia peruensis tiene la capacidad de transmitir también otras especies de Bartonella.

\section{OTROS POTENCIALES VECTORES DE LA ENFERMEDAD DE CARRIÓN EN EL PERÚ}

En el Perú se ha investigado si existen otros artrópodos vectores de Bartonella bacilliformis, pero hasta el momento parece ser que solo los flebotomíneos del género Lutzomyia son los únicos que transmiten la enfermedad de Carrión ${ }^{(75)}$.

Lutzomyia serrana es el potencial vector de la enfermedad de Carrión en el valle del Monzón, provincia de Huamalíes, Huánuco, por evidencias epidemiológicas. Durante los años 1997-1998 ocurrió un importante brote de bartonellosis humana a lo largo del valle, y el estudio entomológico determinó que Lutzomyia serrana constituye el $93 \%$ del total de especies de la fauna flebotomina de la zona ${ }^{(49,55)}$. Esta especie tiene amplia distribución en áreas de selva alta y baja del Perú, habiéndose reportado su presencia en los departamentos de San Martín, Huánuco, Pasco, Ucayali, Cusco, Madre de Dios y Puno (10,22) (Figura 3).

En las provincias de Jaén, San Ignacio (Cajamarca) y Utcubamba (Amazonas), Lutzomyia maranonensis y Lu. robusta son los probables vectores de la enfermedad de Carrión por evidencias epidemiológicas, ya que ambas especies constituyen el $89 \%$ del total de flebotomíneos capturados en áreas donde han ocurrido brotes de esta enfermedad (76) (Figura 3).

Lutzomyia pescei es un potencial vector de la enfermedad de Carrión en las provincias de Huancavelica, Churcampa y Tayacaja (Huancavelica), Urubamba, Calca y Quispicanchis (Cusco), pues ha sido capturada frecuentemente en zonas donde han ocurrido brotes de esta enfermedad ${ }^{(10)}$.

\section{CONCLUSIONES}

La mayor parte de los conocimientos sobre los vectores de la leishmaniasis y la bartonellosis humana en el Perú provienen de estudios en zonas andinas, donde Lutzomyia verrucarum y $L$. peruensis son las especies que presentan más amplia distribución, y además tienen la capacidad de transmitir tanto Leishmania spp. como Bartonella bacilliformis. Otros vectores importantes de leishmaniasis son Lutzomyia tejadai, Lu. ayacuchensis y Lu. pescei. Por primera vez en el Perú, Lutzomyia tejadai ha sido encontrado infectado naturalmente con un híbrido Leishmania braziliensis / Leishmania peruviana.

Se tiene muy pocas referencias de los vectores de la leishmaniasis en la selva amazónica, donde se distribuye el $80 \%$ de especies de flebotomíneos. Recientemente Lutzomyia auraensis fue encontrada infectada naturalmente con Leishmania braziliensis y Leishmania lainsoni en Madre de Dios, lo que constituye un nuevo reporte para la ciencia. Otras especies de potenciales vectores de leishmaniasis en la Amazonía peruana son Lutzomyia yuilli yuilli, Lu. chagasi y Lu. davisi.

La biología molecular promete ser una de las herramientas más útiles para determinar la infección natural de Lutzomyia con Leishmania y Bartonella, dada su gran sensibilidad y especificidad, además que permite el análisis de un gran número de muestras en un corto periodo de tiempo, pero debe ser complementado con el estudio biológico de la transmisión de la enfermedad por el vector a un hospedero susceptible bajo condiciones de laboratorio. EI FRET Nested Real Time PCR permite detectar la presencia de Leishmania en el vector, pero, además, es posible identificar la especie de Leishmania infectante en una sola reacción de PCR, lo que la convierte, junto con el secuenciamiento del $A D N$, en una de las técnicas de mayor impacto en el estudio de los vectores de la leishmaniasis y bartonellosis en el Perú. 
Agradecimientos: Al Doctor Jaime Sánchez Venegas (Facultad de Ciencias Biológicas, Universidad Nacional Mayor de San Marcos) y la Doctora Carmen Flores Mendoza (Centro de Investigación de Enfermedades Tropicales NAMRU-6), por sus acertados comentarios y sugerencias.

Contribución de los autores: VZ y LE participaron en la cconcepción del artículo y la recolección de información, VZ participó en la redacción del artículo; GV, PR y LE participo en la revisión crítica del artículo; GV y PR participaron en la aprobación de la versión final

Fuente de financiamiento: autofinanciado.

Conflictos de interés: los autores declaran no tener ningún conflicto de interés

\section{REFERENCIAS BIBLIOGRÁFICAS}

1. Young D, Duncan M. Guide to the identification and geographic distribution of Lutzomyia sand flies in Mexico, the West Indies, Central and South America (Diptera: Psychodidae). Associated Publishers American Entomological Institute. Memorias of the American Entomological Institute. $1994 ; 54: 1-881 \mathrm{p}$.

2. Munstermann LE. Phlebotomine sand flies. The Psychodidae. In Marquart WD (ed.). Biology of diseases vectors. 2nd. Ed. Elsevier Academic Press, Burlington: 2004; 141-51.

3. Moreno M, Ferro C, RosalesChilama M, Rubiano L, Delgado M, Cossio A et al. First report of Warileya rotundipennis (Psychodidae: Phlebotominae) naturally infected with Leishmania (Viannia) in a focus of cutaneous leishmaniasis in Colombia. Acta Tropica 2015; 148:191-196.

4. Galati E.A. (in Rangel E, Lainson R. 2003) Flebotomineos do Brasil. 2. Morfología y taxonomía. Pag. 23-53. Editora FIOCruz, Fundacao Oswaldo Cruz. Rio de Janeiro, Brasil.

5. Andrade Filho J, Brazil R, Falcao A, Galati E. Description of Pintomyia (Pifanomyia) paleotrichia, a Miocene period new species from the Dominican Republic (Diptera: Psychodidae: Phlebotominae). Mem Inst Oswaldo Cruz. 2007; 102(8):901-3.

6. Ready P. Biology of Phlebotomine sand flies as vectors of disease agents. Annu Rev Entomol. 2013; 58:227-50.

7. Maurin M, Birtles R, Raoult D. Current Knowledge of Bartonella Species. Eur J Clin Microbiol Infect Dis. 1997; 16:487-506.

8. Birtles R, Fry N, Ventosilla P, Caceres A, Sanchez E, Vizcarra H, Raoult D. Identification of Bartonella bacilliformis Genotypes and Their Relevance to Epidemiological Investigations of Human Bartonellosis. J Clin Microbiol. 2002; 40(10):3606-12.
9. Dehio C. Infection-associated type IV secretion systems of Bartonella and their diverse roles in host cell interaction. Cel Microbiol. 2008; 10(8):1591-98.

10. Caceres A, Galati E. Lista de Phlebotominae (Diptera: Psychodidae) para el Perú y especies consideradas como vectores naturales eincriminadas en la transmisión de patógenos de la leishmaniosis tegumentaria y la Enfermedad de Carrión (verruga peruana). Rev Peru Med Exp Salud Pública. 2001; 18(3-4):100-06.

11. Perez E. Studies of Lutzomyia spp. vectors of Leishmaniasis in Perú. Thesis submitted in accordance with the requirements of the University of Liverpool for the degree of Doctor in Philosophy. 1995.233p.

12. Weiss P. Epidemiología y clínica de las leishmaniosis tegumentarias en el Perú. Rev Med Exp. 1943; 2(3):209-48.

13. Weiss P. Las zonas andinas de patología de Phlebotomus. An Fac Med. 1953; 36(1):1-11.

14. Herrer A. Verruga y Uta en el valle de Huaillacayan (Dpto. de Ancash). I. Determinación de los límites altitudinales de la zona endémica y de la incidencia de ambas enfermedades. Rev Peru Med Exp Salud Pública. 1957; 11(1-2):40-49.

15. Blancas F, Caceres A, Galati E. Descricao de Lutzomyia caballeroi, SP.N. (Diptera, Psychodidae, Phlebotominae) Dos Andes Peruanos. Rev Bras Ent. 1989; 33(3/4):455-63

16. Perez E, Villaseca, Llanos A, Campos M, Guerra H. Técnicas para colectar "titiras" (Lutzomyia spp. Diptera: Psychodidae) en ambientes altoandinos peruanos. Rev Per Ent. 1988;29:77-80.http://sisbib.unmsm. edu.pe/BVRevistas/entomologia/v30/ pdf/a19v30.pdf

17. Caceres A. Especies de Lutzomyia (Diptera, Psychodidae, Phlebotominae) vectores de la "uta” en el Perú. Rev Per Ent. 1995;38:23-6.
18. Llanos B, Martins A, Da Silva J. Estudos sobre os Flebotomineos do Peru - II. Departamento de Madre de Dios: Lista das especies coletadas e descricao das femeas da Lutzomyia micropyga (Mangabeira, 1942) e Lutzomyia calcarata Martins E Silva, 1964 (Diptera, Psychodidae, Phlebotominae). Rev Bras Biol. 1976; 36(2):479-85.

19. Llanos B. Flebótomos de la selva peruana (Diptera, Psychodidae). Rev Per Ent. 1973; 16(1):29-49.

20. Perez E, Ogusuku E. Estacionalidad de Lutzomyia spp. (Diptera, Psychodidae) en Coloradito (Pillcopata, Cusco). Rev Per Ent. 1995; 37:89-96.

21. Tejada A. Leishmaniasis tegumentaria en el Perú. Investigación epidemiológicoclínica de la leishmaniasis tegumentaria en los departamentos del Cuzco y Madre de Dios. Tesis de Doctorado. Programa Académico de Medicina Humana. UNMSM. Lima. 1973; 210p.

22. Caceres A, Galati E, Pinto J, Paredes R, Reategui R, Perez J, et al. Psychodidae (Diptera) del Perú I: Phlebotominae en Huánuco, Pasco y Cusco, su relación con la enfermedad de Carrión y la leishmaniosis tegumentaria. Rev Per Biol. 2000; 7(1):27-43.

23. Davies C, Fernandez M, Paz L, Roncal N, Llanos-Cuentas A. Lutzomyia verrucarum can transmit Leishmania peruviana, the aetiological agent of Andean cutaneous leishmaniasis. Trans R Soc Trop Med Hyg. 1993; 87:603-06.

24. Takaoka H, Gomez E, Alexander B, Hashiguchi Y. Natural Infections with Leishmania Promastigotes in Lutzomyia ayacuchensis (Diptera: Psychodidae) in an Andean Focus of Ecuador. J Med Entomol. 1990; 27(4):701-2.

25. Perez E, Veland N, Espinosa D, Torres K, OgusukuE,Llanos-Cuentas A, etal. Isolation and molecular identification of Leishmania (Viannia) peruviana from naturally infected 
Lutzomyia peruensis (Diptera: Psychodidae) in the Peruvian Andes. Mem Inst Oswaldo Cruz. 2007; 102(6):655-58.

26. Volf P, Myskova J. Sand flies and Leishmania: specific versus permissive vectors. Trends in Parasitology. 2007; 23(3):91-92.

27. Guimaraes V, Pruzinova K, Sadlova J, Volfova V, Myskova J, Brandao Fhilo Set al. Lutzomyia migonei is a permissive vector competent for Leishmania infantum. Parasites\&Vectors. 2016; 9:159..

28. Kato $H$, Uezato $H$, Katakura $K$, Calvopiña M, Marco J, Barroso P et al. Detection and identification of Leishmania species within naturally infected sand flies in the Andean areas of Leishmaniasis in Ecuador by a Polymerase Chain Reaction. Am J Trop Med Hyg. 2005; 72(1):87-93.

29. Ministerio de Salud. Boletín Informativo del Perú. 2016; Vol 25, Semana Epidemiológica 51, 18-24 de Diciembre.

30. World Health Organization. Control of the leishmaniases. Report of a meeting of the WHO Expert Committee on the Control of Leishmaniases, Geneva, 22-26 March 2010. 202p.

31. Grimaldi G and Tesh R. Leishmaniases of the New World: current concepts and implications for future research. Clin Microbiol Rev. 1993; 6(3):230-50.

32. Luyo-Acero G, Uezato H, Oshiro M, Takei K, Kariya K, Katakura K, et al. Sequence variation of the Cytochrome $b$ gene of various human infecting members of the genus Leishmania and their phylogeny. Parasitology. 2004; 128:483-91.

33. Lainson R. Ecological interactions in the transmission of the leishmaniases. Phil Trans R Soc Lond B. 1988; 321:389-404.

34. Llanos-Cuentas A, Roncal N, Villaseca P, Paz L, Ogusuku E, Perez E, et al. Natural infections of Leishmania peruviana in animals in the Peruvian Andes. Trans R Soc Trop Med Hyg. 1999; 93(1):15-20.

35. Calvopiña M, Uezato H, Gomez E, Kato $\mathrm{H}$, Mimori T, Nonaka S, Hashiguchi Y. Clinical features, parasites and geographical distribution of American Tegumentary Leishmaniasis in Ecuador. In: Studies on New and Old World Leishmaniases and their transmission, with particular reference to Ecuador, Argentina and Pakistan. Research Report Series No. 7. 2004; p.86-95.

36. Soria J. Leishmaniasis tegumentaria, casos diagnosticados en el Instituto de Medicina
Tropical, 1975-1990. Rev per Med Trop UNMSM. 1993; 7(2):59-80.

37. Alvar J, Vélez I, Bern C, Herrero M, Desjeux P, Cano J et al. Leishmaniasis Worldwide and Global Estimates of Its Incidence. PLoS ONE. 2012; 7(5): e35671. doi:10.1371/journal.pone. 0035671

38. Villaseca P, Llanos-Cuentas A, Pérez E, Davies C. A comparative field study of the relative importance of Lutzomyia peruensis and Lutzomyia verrucarum as vectors of cutaneus leishmaniasis in the Peruvian Andes. Am J Trop Med Hyg. 1993; 49(2):260-69.

39. Herrer A. Estudios de Leishmaniasis Tegumentaria en el Perú. VI. Relación entre Leishmaniasis Tegumentaria y Phlebotomus. Rev Per Med Exp Salud Pública. 1951; 8: 119-137.

40. Lainson R. The American leishmaniases: some observations on their ecology and Epidemiology. Trans R Soc Trop Med Hyg. 1983; 77(5):569-96.

41. Hashiguchi Y, Gomez E. A review on andean leishmaniasis. In: Studies on New World Leishmaniasis and its Transmission, with Particular Reference to Ecuador. 1992; 182p.

42. Lucas C, Franke E, Cachay M, Tejada A, Cruz M, Kreutzer R, et al. Geographic distribution and clinical description of leishmaniasis cases in Peru. Am J Trop Med Hyg. 1998; 59(2): 312-17.

43. Kato H, Caceres A, Mimori T, Ishimaru Y, Sayed A, Fujita M. et al. Use of FTA Cards for Direct Sampling of Patients' Lesions in the Ecological Study of Cutaneous Leishmaniasis. J Clin Microbiol. 2010; 48(10):3661-65.

44. Tejada A, Tejada O, Zorrilla V. Leishmaniasis tegumentaria en Ambo, Huánuco. Rev per Med Trop UNMSM. 2001; 1(1):21-28.

45. Dujardin JC, Bañuls A, Llanos-Cuentas A, Alvarez E, DeDoncker S, Jacquet D et al. Putative Leishmania hybrids in the Eastern Andean valley of Huanuco, Peru. Acta Tropica. 1995; 59:293-307.

46. Kato H, Cáceres A, Hashiguchi Y. First evidence of a Hybrid of Leishmania (Viannia) braziliensis/L.(V.) peruviana DNA detected from the Phlebotomine sand fly Lutzomyia tejadai in Peru. PLoS Negl Trop Dis 10(1): e0004336. doi:10.1371/journal.pntd.0004336.
47. Maguiña C, Ugarte C, Breña P, Ordaya E, Ventosilla P, Huarcaya E, et al. 2008. Actualización de la Enfermedad de Carrión. Rev Med Hered. 2008; 19(1):36-41.

48. Pachas P. Enfermedad de Carrión (bartonelosis) en el Perú. Ministerio de Salud, Oficina General de Epidemiología. 2001; 88 p.

49. Tejada A, Vizcarra H, Perez J, Caceres A, Quispe J, Pinto J et al. Estudio Clínico epidemiológico de bartonellosis humana en el valle del Monzón, Huamalíes, Huánuco. An Fac Med UNMSM. 2003; 64(4):211-17.

50. Ellis B, Rotz L, Leake J, Salmavides F, Bernable J, Ventura G et al. An outbreak of acute bartonellosis (Oroya fever) in the Urubamba region of Peru, 1998. Am J Trop Med Hyg. 1999; 6(12):344-49.

51. Cuadra M, Cuadra A. Enfermedad de Carrión: Inoculaciones de Seres Humanos con Bartonella bacilliformis, Una Revisión. An Fac Med UNMSM. 2000;61(4):289-94.

52. Young D, Arias J. Phlebotominae sand flies in the Americas. Pan American Health Organization. Technical paper No. 33. $1991 ; 27 \mathrm{p}$.

53. Hertig M. Sand flies of the genus Phlebotomus: A review of their habits, diseases relationships, and control. In: The proceedings of the fourth International Congresses on Tropical Medicine and Malaria. May 10-18 1948; p.1609-15.

54. Ogusuku E, Perez E. Nota Científica: Duración de los estadíos inmaduros de Lutzomyia peruensis y Lutzomyia verrucarum (Diptera, Psychodidae, Phlebotominae) del Perú. Rev per Ent. 1996; 38:27-28.

55. Tejada A, Cáceres A, Zorrilla V, Palacios O, Miranda J. Distribución de Lutzomyia (Diptera: Psychodidae: Phlebotominae) en el valle del Huallaga, Huánuco. Rev per Med Trop. 2004; 9(1): 9-18.

56. Caceres A, Villaseca P, Dujardin J, Bañuls A, Inga R, Lopez M, et al. Epidemiology of Andean cutaneous leishmaniasis: incrimination of Lutzomyia ayacuchensis (Diptera: Psychodidae) as a vector of Leishmania in geographically isolated, upland valleys of Peru. Am J Trop Med Hyg. 2004; 70(6):607-12.

57. Córdova O, Vargas F, Hashiguchi Y, Kato $\mathrm{H}$, Gómez E. Identificación de especies de Leishmania en pacientes y flebotominos en áreas de transmisión de una región del 
Perú. Rev Peru Med Exp Salud Pública. 2011;28(3):446-53.

58. Fraiha H, Quintana J, Mendes de Souza M. Consideracoes entomo-epidemiologicas sobre a fauna de flebótomos de Iquitos, Peru (Diptera, Psychodidade, Phlebotominae). Revista da FSESP. 1980; 25(1/80):20-23.

59. Páucar R. Estudio de especies del género Lutzomyia como posibles vectores de Leishmania en el distrito de Echarati, provincia de La Convención, Cusco. Tesis para optar título profesional de biólogo. Facultad de Ciencias Biológicas, Universidad San Antonio de Abad, Cusco. 2001

60. Valdivia H, De los Santos M, Fernandez R, Baldeviano C, Zorrilla V, Vera $\mathrm{H}$ et al. Natural Leishmania Infection of Lutzomyia auraensis in Madre de Dios, Peru, detected by a Fluorescence Resonance Energy Transfer-Based RealTime Polymerase Chain Reaction. Am J Trop Med Hyg. 2012; 87(3):511-17.

61. Galati E, Caceres A, Le Pont F. Description of Lutzomyia (Pifanomyia) robusta n. sp. (Diptera, Psychodidae, Phlebotominae) from Peruvian Ecuadorean interandean areas. Rev Saude Pública. 1995; 29(2): 89-99.

62. Herrer A. Lutzomyia peruensis (Shannon 1929) posible vector de la "uta" (leishmaniasis tegumentaria). Rev Inst Med Trop Sao Paulo. 1982; 24(3):168-72.

63. Lanzaro G, Warburg A. Genetic variability in phlebotomine sand flies: possible implications for leishmaniasis epidemiology. Parasitology today. 1995; 11(4):151-54.

64. Lopez $\mathrm{M}$, Inga $\mathrm{R}$, Cangalaya $\mathrm{M}$, Echevarria J, Llanos-Cuentas A, Orrego
C, et al. Diagnosis of Leishmania using the polymerase chain reaction: a simplified procedure for field work. Am J Trop Med Hyg. 1993; 49(3):348-56.

65. Perez E, Ogusuku E, Inga R, Lopez M, Monje J, Paz L, et al. Natural Leishmania infection of Lutzomyia spp. in Peru. Trans R Soc Trop Med Hyg. 1994; 88:161-64.

66. Kato H, Caceres A, Gomez E, Mimori T, Uezato H, Marco J, et al. Short Report: Molecular Mass Screening to Incriminate Sand Fly Vectors of Andean-type Cutaneous Leishmaniasis in Ecuador and Peru. Am J Trop Med Hyg. 2008; 79(5): 719-21.

67. Kato H, Uezato H, Gomez E, Terayama Y, Calvopiña M, Iwata H et al. Establishment of a Mass Screening Method of Sand Fly Vectors for Leishmania Infection by Molecular Biological Methods. Am. J. Trop. Med. Hyg. 2007; 77(2):324-329.

68. Kato H, Gomez E, Caceres A, Vargas F, Mimori T, Yamamoto $\mathrm{K}$ et al. Natural Infections of Man-Biting Sand Flies by Leishmania and Trypanosoma Species in the Northern Peruvian Andes. Vector Borne and Zoonotic Diseases. 2011; 11(5):515-521.

69. Pérez E, Ogusuku E. Notas sobre la descripción de Lutzomyia verrucarum (Townsend, 1913) (Diptera, Psychodidae). Rev Per Ent. 1995; 37:127-28.

70. Caceres A. Distribución geográfica de Lutzomyia verrucarum (Townsend, 1913) (Diptera, Psychodidadae, Phlebotominae) Vector de la Bartonellosis Humana en el Perú. Rev Inst Med Trop Sao Paulo. 1993; 35(6):485-90.

71. Ponce C, Solórzano N. Evaluación de la transmisión vertical de Bartonella bacilliformis en Lutzomyia verrucarum
(Diptera: Psychodidae). Rev Peru Med Exp Salud Pública. 2002; 19(2):93-6.

72. Romero S. Detection of Bartonella bacilliformis by Real Time PCR in naturally infected sand flies. Thesis Master ofScience. USUHS. 2004; 83 p. www.dtic. $\mathrm{mil} /$ get-tr-doc/pdf ?AD=ADA 434804

73. Norman A, Regnery R, Jameson P, Greene C, Krause D. Differentiation of Bartonella-Like isolates at the species level by PCR-Restriction Fragment Length Polymorphism in the Citrate Synthase Gene. J Clin Microbiol. 1995; 33(7):1797-1803.

74. Villaseca P, Padilla C, Ventura G, Samalvides F, Yañez H, Chevarría L, et al. Importancia de la Lutzomyia peruensis en la transmisión de la Enfermedad de Carrión en el Valle Sagrado de los Incas. Urubamba-Cusco, Perú. Rev Peru Med Exp Salud Pública. 1999; 15(1-2):28-30.

75. Billeter S, Caceres A, Gonzales J, Luna D, Kosoy M. Molecular detection of Bartonella species in ticks from Peru. J Med Entomol. 2011; 48(6):1257-60.

76. Caceres A, Galati E, Le Pont F, Velásquez C. Possible role of Lutzomyia maranonensis and Lutzomyia robusta (Diptera: Psychodidae) as vectors of human bartonellosis in three provinces of Región Nor Oriental del Marañón, Perú. Rev Inst Med Trop Sao Paulo. 1997; 39(1):51-2.

Correspondencia: Victor Zorrilla

Dirección: Departamento de Entomologia, Centro de Investigación de Enfermedades Tropicales de la Marina de los EE.UU. NAMRU-6. Avenida Venezuela cdra. $36 \mathrm{~s} / \mathrm{n}$, Bellavista, Callao (Centro Médico Naval). Perú. Teléfono: 6144118, 972678689

Correo electrónico:victorzorrilla01@gmail.com

\section{Nuestros artículos se encuentran indizados en:}

$$
\text { PublMed }
$$

